\title{
II \\ Cerámicas del siglo XVIII en la Isla de Pascua, Rapa Nui
}

\author{
Daniel Schávelzon, Flavia Zorzi y Ana Igareta
}

\section{Resumen}

El presente texto reporta el hallazgo casual de cuatro fragmentos de cerámica en el Ahu Tahai, en la costa oeste de la Isla de Pascua. Los mismos eran parte de una taza o pequeña vasija y sus rasgos son típicos de la cerámica hispano-americana de los siglos XVII y XVIII. La presencia de cerámica en Rapa Nui es exótica y hasta donde sabemos jamás han sido reportados allí hallazgos históricos de estas características.

\section{Abstract}

The casual discovery of four fragments of pottery in the Ahu Tahai on the west coast of Easter Island is reported. They were part of a cup or small bowl and its features are typical of the Spanish-American ceramics of the seventeenth and eighteenth centuries. The presence of pottery in Rapa Nui is exotic and as far as we know there had never been reported historical finds like this one.

\section{El hallazgo}

El estudio de la cerámica arqueológica de la Isla de Pascua parece estar signado por el engaño que sufriera la expedición de Thor Heyerdahl en $1955^{1}$, cuando el intento de hacer pasar unas cerámicas como provenientes de un hallazgo pre-Contacto europeo dejó su impronta. Encontrar cerámica arqueológica en Pascua -incluso posterior al Contacto- implica superar las barreras creadas por aquel intento de estafa. Y si bien somos conscientes del peso de ese legado, no podemos por ello dejar de referir un reciente hallazgo.

En la entrada de una las viviendas reconstruidas en el Ahu Tahai se encontraron cuatro fragmentos cerámicos de posible origen colonial americano que habían sido desenterrados debido al constante pisoteo al que este lugar se ve sometido a diario. El material fue colectado y entregado al Museo Padre Sebastián Englert de la isla para su preservación. Hasta donde sabemos este es el primer hallazgo de cerámica colonial americana que se reporta en la isla, fuera del caso insólito narrado al inicio. Se desconoce si en el sitio pudiera haber otros fragmentos de la misma u otra cerámica bajo el nivel superficial ya que sería necesaria una excavación para determinarlo. Y aunque después lo analizamos Heyerdhal cita en diversas ocasiones en sus excavaciones en cuevas la presencia de material cerámico, pero nada dice de ello que nos sea útil.

El lugar de colecta es la pequeña entrada a una de las construcciones que, unidas a cuevas posteriores, debieron servir de vivienda. Sabemos que estas estructuras fueron rehechas por William Mulloy y el Padre Englert en $1974^{2}$, sin dejar evidencias que permitieran distinguir entre lo nuevo y lo viejo tal como lo indica hoy la normativa internacional. En el caso de la estructura donde se hallaron las cerámicas, puede verse a simple vista que se trata de un aparejo de piedra rehecho, si bien sabemos por los trabajos arqueológicos desarrollados en el sitio que es muy posible que la reconstrucción haya sido fidedigna a las

\footnotetext{
${ }^{1}$ Carlyle Smith, A small pottery scan, Rapa Nui Journal, vol. II, no. 3, pp. 4-5, 1988.

2 William T. Mulloy, The Easter Island Bulletins of William Mulloy, World Monuments Fund \& Easter Island Foundation, PP. 55-63, Nueva York, 1995; William T. Mulloy y S. R. Fischer, Easter Island Studies: Contributions to the History of Rapa Nui in Memory of William T. Mulloy, Oxbow Books, Oxford, 1993.
} 
evidencias materiales, y eso parece indicar la trinchera excavada por W. S. Ayres ${ }^{3}$.

En tal sentido, y si bien aquí no hubo control arqueológico y por ende cualquier interpretación es ligera, el lugar en que se lo realizó y las características de los materiales le otorgan relevancia y permiten plantear interesantes preguntas.

\section{Descripción de la cerámica}

Se trata de cuatro tiestos cerámicos correspondientes a una o dos piezas de elaboración manual, cubiertos en ambas superficies por pintura roja pulida. Uno de ellos es parte de un asa de sección circular, mientras que los otros tres se ensamblan entre sí y corresponden al borde de un recipiente abierto con labio convexo. La pasta es de grano bastante grueso, porosa, de fractura irregular, cocción oxidante completa y color ante. Presenta inclusiones visibles a ojo desnudo.

Por el tratamiento de superficie -así como por las formas- esta cerámica recuerda a piezas halladas en distintas zonas de la América hispana en contextos de los siglos XVII y XVIII. Nos referimos a un grupo bastante diverso de cerámicas de elaboración manual con pintura roja pulida o bruñida, denominada de distinta manera según el área de hallazgo y las características específicas que exhiben a nivel local. Se las conoce como Cerámica Roja Fina en Panamá ${ }^{4}$, cerámica Monocroma $R_{\text {Roja }}^{5}$ y Candelaria Rojo ${ }^{6}$ en Argentina, Mexican Red Painted Earthenware ${ }^{7}$ y Cerámica Roja Bruñida Colonial ${ }^{8}$ en México, Terracota Fina en Ecuador ${ }^{9}$, entre otras denominaciones. También fueron identificadas en $\mathrm{Chile}^{10}$, en Colombia ${ }^{11}$ y en otras áreas de la América hispana.

El desarrollo de este tipo de cerámica ha sido interpretado como producto del contacto entre tradiciones cerámicas indígenas y europeas ${ }^{12}$ y con toda seguridad han sido elaboradas simultáneamente en varios puntos del imperio español. ${ }^{13}$

En algunos casos, como en el área del Río de la Plata, las piezas rojas pulidas presentan formas derivadas de la tradición ibérica (jarras, cantimploras, platos, bacines, candeleros) inexistentes en la región antes de

\footnotetext{
3 William Ayres, The Tahai settlement complex, First International Congress, Eastern Island and the East Pacific, (C. Cristino, P. Vargas, R. Izaureta y R. Buddd editors), Universidad de Chile, Vol. I, pp. 105-119, Santiago, 1988.

${ }^{4}$ Beatriz Rovira y Felipe Gaitán, Los búcaros. De las Indias para el mundo, Canto Rodado, Vol. V, pp. 41-80, 2010.

${ }^{5}$ Daniel Schávelzon, Catálogo de cerámicas históricas de Buenos Aires (siglos XVI-XX). Con notas sobre la región del Río de la Plata, Centro de Arqueología Urbana, Buenos Aires, publicación en CD, Centro de Arqueología Urbana, Buenos Aires, 2001.

${ }^{6}$ Beatriz Rovira, La arqueología histórica del conjunto jesuítico de La Candelaria (provincia de Misiones, Argentina), Tesis doctoral presentada a la Universidad Nacional de La Plata, 1989.

${ }^{7}$ Hale Smith, Two historical archaeological sites in Brevard County, Florida, Florida Anthropological Society Special Publication, Vol. I, Florida, 1949; Kathleen Deagan, Artifacts of the Spanish colonies of Florida and the Caribbean 1500-1800, Smithsonian Institution Press, Washington, 1987, pp. 43-44.

${ }^{8}$ T. Charlton, P. Fournier y J. Cervantes, La cerámica del periodo colonial temprano en Tlatelolco: el caso de la loza roja bruñida, Presencias y encuentros. Investigaciones arqueológicas de salvamento, Instituto Nacional de Antropología e Historia, México, 1995 , pp. 135-155.

9 Jozef Buys, Monumentos y fragmentos: Arqueología Histórica en el Ecuador, Approaches to the Historical Archaeology of México, Central and South América, (J. Gasco, G. Ch. Smith y P. Fournier-García edits.), The Institute of Archaeology, University of California, Los Angeles, 1997, pp. 111-119.

${ }^{10}$ Claudia Prado Berlien, Precisiones en relación a un tipo cerámico característico de contextos urbanos coloniales en la zona central de Chile, Actas del XVII Congreso de Arqueología Chilena, Valdivia, 2010, Vol. II, pp. 1011-1024.

${ }^{11}$ Mónica Therrien, Elena Uprimny, Jimena Lobo Guerrero, María Salamanca, Felipe Gaitán y Marta Fandiño, Catálogo de cerámica colonial y republicana de la Nueva Granada: producción local y materiales foráneos (Costa Caribe, Altiplano Cundiboyacense, Colombia), Fundación de Investigaciones Arqueológicas Nacionales, Bogotá, 2002.

${ }^{12}$ Carlos Ceruti y Nora Natassi, Evidencias del contacto hispano-indígena en la cerámica de Santa Fe la Vieja (Cayastá), Revista del Museo de Historia Natural de San Rafael (Mendoza), Vol. IV, pp. 213-136, 1977.

Th. Charlton, Fournier \& Cervantes, op. cit. p.148; Schávelzon, op. cit.; Rovira y Gaitán, op. cit., 47; Flavia Zorzi y Agustín Agnolin, Análisis y reflexiones en torno a un conjunto cerámico colonial en la ciudad de Buenos Aires, Cuadernos del Instituto Nacional de Antropología y Pensamiento Latinoamericano, 2013), Vol. I, no. 3, pp. 132-141.

13 Rovira y Gaitán, op. cit., 62-63.
} 
la llegada de los españoles. ${ }^{14}$ La pintura roja puede presentarse cubriendo la totalidad de las superficies o disponiéndose en líneas bordeando la boca, así como en líneas transversales o pinceladas expeditivas. A veces, el pulido se concentra exclusivamente en las superficies pintadas.

En muchos casos, estas cerámicas rojas americanas parecen emular las formas y decoraciones de la cerámica roja conocida en la arqueología como Mérida ${ }^{15}$ y Micácea Naranja ${ }^{16}$, producida en Estremoz y otras áreas de Portugal y España desde el siglo XVI. ${ }^{17}$ Se cree que esas cerámicas, representadas por jarras, cuencos, escudillas, pocillos y otros recipientes, derivaron a su vez de la imitación del trabajo en metal, razón por la cual suelen presentar decoración moldeada imitando el repujado ${ }^{18} \mathrm{y}$, en algunos casos, tienen gran cantidad de mica o un fuerte bruñido, lo que ha sido interpretado como un intento de imitar el brillo metálico ${ }^{19}$.
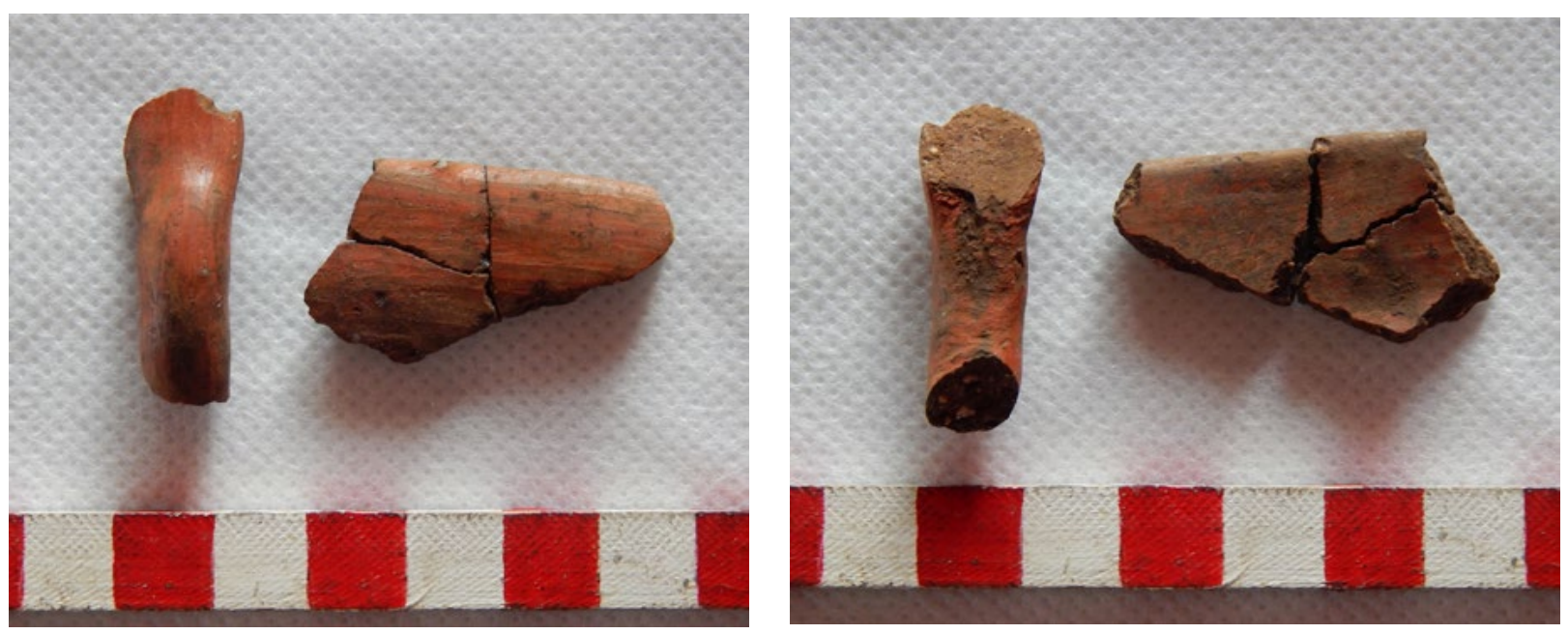

FIGS. 8-9 VISTA ANTERIOR Y POSTERIOR DE LOS FRAGMENTOS DE CERÁMICA.

\section{La interpretación}

Investigaciones previas han establecido que en Pascua no se hizo cerámica ni se la trajo de fuera durante los siglos de desarrollo de la cultura rapanui. Sí hubo cerámica en Oceanía mucho antes de que iniciara el poblamiento de la isla; se trata de la llamada cerámica Lapita, pero no hay posibilidad de una confusión entre dicha cerámica y la aquí reportada. ${ }^{20}$

Los fragmentos hallados corresponden probablemente a una misma pieza, una vasija utilitaria simple y

\footnotetext{
${ }^{14}$ Ceruti y Natassi, op.cit., pag. 222; Schávelzon 2001, op.cit.; Zorzi y Agnolín, op.cit., 138.

15 John Hurst, David Neal y Hendrik Jan Van Beuningen, Pottery produced and traded in North-West Europe 1350-1650, Rotterdam papers vol. VI, Gepubliceerd door, Rotterdam, 1987.

16 Bruce Council, Archaeology of the Convento de San Francisco, Tesis de Maestría presentada en la Universidad de Florida, Departamento de Antropología, 1975; Deagan, (op.cit.), 40-41.

17 Rovira y Gaitán, (op. cit.), 59-61; Antonio Rodríguez Santamaría y Enrique Moraleda Olivares, La cerámica bucarina en Talavera de la Reina (siglos XVI-XVII), Cuaderna no. 5, 1997, pp. 21-35; Alejandra Gutiérrez, Portuguese coarsewares in early modern England: reflections on an exceptional pottery assemblage from Southampton, Post-Medieval Archaeology Vol. XXXXI, no. 1,2007 , pp. 64-79.

${ }^{18}$ Alfonso Pleguezuelo, Cerámicas para el agua en el Barroco español: una primera aproximación desde la literatura y la pintura, Ars Longa Vols. IX-X, 2000, pp. 123-138.

19 Idem, pag. 134.

20 Patrick Vinton Kirch, The Lapita Peoples: Ancestors of the Oceanic World, Blackwell, Cambridge, 1997; On the Road of the Winds: An Archaeological History of the Pacific Islands before European Contact, University of California Press, Berkeley, 2000.
} 
modesta. Pudo llegar con cualquiera de las expediciones arribadas a Pascua en algún momento desde el Contacto en adelante, pero su similitud morfológica y estilística con piezas americanas coloniales nos lleva a asociar su presencia con la de los viajeros que pasaron por el continente americano o salieron desde allí hacia la isla durante dicho período.

El primero de ellos, al menos entre los pocos de los que hay documentación fidedigna, fue Phelipe González de Haedo, quien emprendió su viaje desde el continente en 1770. Las diferentes versiones escritas acerca de esa expedición indican que Haedo estuvo en lo que hoy es Hanga Roa, en las cercanías del Ahu Tahai. El mapa y la descripción escrita que registran los hechos de la expedición indican la existencia en el lugar de moais erguidos, por lo que es posible deducir que su arquitectura asociada se encontraba aún en uso ${ }^{21}$. Quizás una de las casas entonces visitadas por los viajeros haya estado próxima a las hoy reconstruidas, o ahí quedó entero o fragmentado uno de los objetos intercambiados entre los viajeros y la población local que aún usaba las casas tradicionales. Luego viajaron a la isla el barco Volador, en una expedición organizada por Jacques A. Moerenhout, que en enero de 1828 estuvo pocos días, información que sólo recientemente ha salido a la luz ${ }^{22}$. Más tarde viajó otro barco que se ha mantenido en el anonimato por sucesos históricos muy específicos -lo secreto de la misión- y fue la goleta Colo Colo bajo el capitán Leoncio Señoret Montagna que arribó al lugar en 1837 y 1850; y es posible que haya habido un tercer barco chileno en 1842.

En 1868, pasó por la isla la sí reconocida por la historiografía tripulación de la Topaze, que zarpó del puerto peruano del Callao y relató la existencia de casas en uso en el lugar. También los misioneros cristianos que se instalaron en la Isla en la década de 1860 tuvieron un contacto cada vez más frecuente con el continente, tanto con Chile como con Perú.

\section{Repensando el engaño a Heyerdahl}

En su viaje de 1955, el explorador noruego Thor Heyerdahl sufrió un engaño que dejó su marca en la arqueología de Pascua. ${ }^{23}$ Heyerdahl estaba obsesionado por hallar cerámica continental prehistórica, ya que eso cerraría su hipótesis del poblamiento de la Polinesia por pueblos de América del Sur. En su afán, llegó incluso a ofrecer dinero a quien pudiera encontrar cerámica en la Isla de Pascua. Ese ofrecimiento fue precisamente lo que generó el intento de engañarlo. Individuos inescrupulosos quebraron y enterraron entonces una vasija en una casa de Orongo para que fuera descubierta por los expedicionarios. Al releer la información de la época, resulta que la vasija era "an old pot of unknown origin", la que según asumen habría sido elaborada poco antes en un pueblo de Chile donde subsistía aún ese tipo de artesanía. ${ }^{24}$

En el contexto del año 1955, la arqueología de post-Contacto -o arqueología histórica como se la llama genéricamente-, no existía como entidad en el campo de la investigación y no se consideraba que los hallazgos de dicho período pudieran tener importancia frente a lo más antiguo. Es decir que una vez identificada como no-prehistórica nadie estudió la vasija de Heyerdahl y dado que entonces poco o nada se sabía sobre la cerámica histórica de Chile, nadie pudo dar una opinión consistente. El que se la considerara proveniente de una aldea remota que mantenía tradiciones alfareras suena poético, infantil, pero no académico. Nadie podía demostrar que hubiera sido hecha recientemente y menos en una aldea remota, o en donde sobrevivían viejas tradiciones y que luego fuese llevada a la isla. La interpretación científica para descartarla resultaba casi tan absurda como el intento de engaño.

Durante esas mismas excavaciones se encontraron también otras cerámicas históricas, muchas,

${ }^{21}$ Rolf Foester, Rapa Nui, Las primeras expediciones europeas, Rapa Nui Press, Hanga Roa, 2001, pag. 141.

22 Marcos Moncada Astudillo, La tradición naval respecto del primer buque chileno en la Isla de Pascua, Revistamar vol. I, pp. 62-712, 2008.

${ }^{23}$ C. Smith (op.cit.), pag. 5.

${ }^{24}$ C. Smith (op. cit.), pag. 4. 
específicamente lozas. Estas fueron usadas para fechar los estratos como pos-Contacto pero sin dar mayores detalles sobre ellas. Es cierto que en ese momento lo que hoy llamamos "loza" no se podía fechar con precisión, ni estaban establecidas las tipologías siquiera básicas lo que no era un error de los arqueólogos. Valga el caso del sitio de la casa Maunga Auhepa donde había cinco fragmentos de "White, glazed crokery", lo que hoy no quiere decir nada, ya que la loza blanca -la definen como parte de una tazase comenzó a producir en el siglo XVIII y continúa siendo fabricada hasta hoy. ${ }^{25} \mathrm{Si}$ hubiera sido eso, pero lo que hoy llamamos Creamware bien lo pudieron llevar los primeros europeos que llegaron a la isla, lo que hubiera sido más que interesante. En la actualidad, sería impensable dar esa descripción.

Tampoco los vidrios hallados fueron siquiera descritos o se consideró que podrían tener diferentes antigüedades y que bien podrían haber sido del siglo XVIII. Y esto sucedió en varias ocasiones con objetos históricos que, a sus ojos, sólo indicaban ser pos-Contacto, lo que no deja de ser cierto. Para que haya algo no pasquense debía ser por fuerza posterior a algún contacto. En el caso citado antes encontraron un problema, una cuenta de vidrio estratigráficamente ubicada en un nivel más antiguo, lo que los llevó a acercarse a la hipótesis de presencias europeas más antiguas que las establecidas y consensuadas. En síntesis, sabemos que sí se han encontrado cerámicas no locales y por su descripción las identificamos como lozas y por ende pos ca. 1760, pero no hay una ilustración para entenderlas.

Lo que pareciera es que nunca antes del hallazgo reportado en este trabajo se había identificado cerámica de pasta roja que hubiese sido descrita como Earthenware. Y a nadie se le había ocurrido hacer arqueología del Contacto en sí mismo, no descartar por ser pos-Contacto. Y menos aun que eso sirviera para explicar la confusa historia de lo sucedido en esos años iniciales de las relaciones con el exterior a la isla.

Esto nos lleva a pensar en la necesidad de buscar sistemáticamente restos del Contacto o post-Contacto y estudiarlos de manera conveniente, sin limitar la arqueología de Rapa Nui al peso de su pasado previo. En particular, sería interesante observar si hay materiales pre-1722, fecha del primer contacto europeo bien documentado con la isla, lo que sería de gran relevancia para la construcción de su historia.

\section{Consideración final}

El pequeño hallazgo del que se da noticia en este texto, sumado a la observación de restos de lozas, anclas y vidrios históricos en diferentes lugares de la Isla de Pascua, y a lo poco descrito del siglo XVIII, indican que las expediciones europeas que llegaron al lugar desde el viaje de descubrimiento de Jacob Roggeveen, y quizás alguna anterior no bien documentada y muy posible, dejaron evidencias materiales del contacto. Pero no nos consta documentalmente que antes de Haedo en 1770 haya habido quienes llegaran a la isla pasando previamente por las costas de Chile, origen de estas cerámicas.

Los materiales cerámicos pueden decir mucho sobre los intercambios y las complejas relaciones que se desarrollaron entre la población local y los marineros de diversos países europeos, así como acerca de los usos que los rapanui le dieron a los nuevos objetos.

La arqueología del Contacto, e incluso de post-Contacto, es una veta poco explorada en la Isla de Pascua y si bien se ha avanzado en estudios de su ocupación en el siglo XIX, tanto en arquitectura como en otro tipo de materiales ${ }^{26}$, la cerámica, el metal y el vidrio de épocas históricas previas siguen esperando un estudio sistemático.

\footnotetext{
${ }^{25}$ Carlyle Smith, The Maunga Auhepa Hose Site, Archaeology of Easter Island, (T. Heyerdahl y E. Ferdon edits.), Monographs of The School of American Research, Albuquerque, 1961, Vol. XXIV, no. 1, pp. 283.

26 Cristian Moreno Pakarati, La importancia de la arqueología histórica en Rapa Nui: el caso de la explotación ovejera y la domesticación del poder colonial, Rapa Nui y la Compañía Explotadora, editor M. Fuentes, Consejo Nacional de la Cultura y las Artes, Valparaíso, 2012, pp. 284-293; Miguel Fuentes, Héctor Orellana y Francisco Rivera, Registro arquitectónico y espacial del sistema de pircas de la Compañía Explotadora de la Isla de Pascua, Rapa Nui y la Compañía Explotadora, editor M. Fuentes, Valparaíso, Consejo Nacional de la Cultura y las Artes, Valparaíso, 2012, pp. 156-191.
} 
EASTER ISLAND ARCHAEOLOGY / ARCHEOLOGICA EN RAPA NUI

\section{Agradecimientos}

Dejamos presente la recepción de la cerámica en el Museo Englert por Francisco Torres Hochstetter y Paula Valenzuela y agradecemos sus amigables expresiones. 